\title{
B-factor analysis suggests that L-lysine and R, R-bisLysine allosterically inhibit Cj.DHDPS Enzyme by decreasing its protein dynamics
}

\author{
S Saran ${ }^{1}$ D A R Sanders ${ }^{1}$ \\ ${ }^{1}$ Department of Chemistry, The University of Saskatchewan \\ sas966@mail.usask.ca
}

In Campylobacter jejuni (Cj.), dihydrodipicolinate synthase (DHDPS) catalyzes the condensation of pyruvate (pyr) and (S)-aspartate- $\beta$-semi-aldehyde (ASA) to form dihydrodipicolinate. Cj.DHDPS regulates an essential step in the biosynthesis of L-lysine (Lys) and meso-diaminopimelate in bacterial cell wall synthesis. In our study, the normalized B-factors calculated from crystallographic data suggested that Lys and synthetic bisLys inhibitors allosterically inhibit the enzyme by reducing mobility in the regions that undergo transient conformational fluctuations to facilitate the diffusion of substrate Pyr in the active site for catalysis, consistent with published HDX-MS dynamics studies. The normalized B-factors also showed that bisLys allosterically inhibits DHDPS Y110F lysine insensitive mutant by rigidifying these flexible regions. The B-factor analysis suggest that flexibility in the regions ( $\beta 8, \alpha 9, \alpha 10, \alpha 11, \mathrm{~L} 20$, and $\alpha 12)$ may be vital for substrate turn over and catalysis. Normalized Bfactors of DHDPS and its Y110F mutant with and without inhibitors in different space groups showed similar flexibility patterns, suggesting the reliability of our B-factors analysis.

Acta Cryst. (2020). A76, a186 